\title{
THE IMPACT OF INFORMATION SHARING ON CONGESTION USING AGENT-BASED SIMULATION
}

\author{
Zheng, W. M.; Jin, M. Z. \& Ren, P. Y. \\ Business School of Sichuan University, Chengdu, 610065, P. R. China \\ E-Mail: jinmaozhu@scu.edu.cn ("Corresponding author)
}

\begin{abstract}
The number of tourists to recreation areas has increased dramatically, leading to a growing concern about the congestion phenomena in these areas. Since the information provision has been introduced to reduce the congestion, its potential benefits as well as its drawbacks have been discussed controversially. In this work, we address a basic recreation area scenario with different patterns of information sharing and study the impact of them using agent-based simulation, where the tourists are modelled as agents. Three evaluation indicators are proposed to evaluate the performance of the strategies and both of the positive and negative of information sharing patterns are described.

(Received, processed and accepted by the Chinese Representative Office.)
\end{abstract}

Key Words: Recreation Areas, Congestion, Information Sharing, Multi-Agent Simulation

\section{INTRODUCTION}

Congestion in recreation areas (e.g., Theme Parks, World Heritage Sites, etc.) has been pointed out as one of the main negative impacts of tourism, especially in some developing areas like China $[1,2]$ because of the centralized of public holidays and huge population. In the context of recreation, congestion has occurred when either the utility of an individual diminishes due to the presence or behaviour of other tourists, or when the costs of doing an activity increase due to the presence of other tourists (costs can be in loss of time, or in actual monetary loss) [3]. It could reduce the time available for participation in tourism activities and could be perceived as an unsatisfactory experience by tourists, having a negative effect on a possible future visit $[4,5]$.

In recent years, special attentions are paid to how to alleviate the congestion through providing recommendation information relative to the areas (i.e. the tour route and popular attractions during the areas; the congestion information regarding each attraction, etc.). Tsai and Chung [6] and Kawamura et al. [7] studied a personalized route recommendation service based on the congestion information and the preference and restrictions of tourists.

However, since the information provision has been introduced to reduce the congestion, its potential benefits as well as its drawbacks have been discussed controversially [8]. Some phenomena or behaviour observed in traffic areas may reflect the negative impacts of the given information [9], Ben-Akiva et al. listed the three phenomena that disprove the benefits of information sharing: oversaturation, overreaction and concentration [10]. In the field of tourism, some studies also revealed the drawbacks of information provision $[11,12]$, such as it may lead to concentration if too many tourists sharing the same information, besides, the time delay of information may causes the congestion to oscillate.

Therefore, understanding the impact of different information is critical to the design and implementation of effective information sharing patterns [13]. There has been little research regarding the systematic ways to of information provision, this work seeks to fill this gap in research by investigating effective ways to provide information for relieving congestion in recreation areas. A basic recreation area scenario with three patterns of information sharing is addressed and the impact of information provision is studied using agent-based simulation. 
Three evaluation indicators are proposed to evaluate the performance of the strategies and both of the positive and negative of information sharing patterns are described.

The remainder of this paper is structured as follows: Some basic notions of agent-based simulation and the behaviour characteristics of agents are sketched in the next section. After that, section 3 illustrates mathematical definition, the simulation experiment design and three information provision strategies. The results of simulation experiments are presented and discussed in detail in section 4. It closes with a summary and an outlook to future work.

\section{AGENT-BASED SIMULATION IN TOURISM DOMAIN}

Recreation areas are complex systems, which comprised of hundreds of acres, moreover, these complex systems often behave nonlinearly and uninterruptedly [14]. Given the complexity of social, environmental and economic interactions, recreation managers need tools that provide insights into the cause and effect relationships between management actions and social and environmental outcomes [15]. In recent years, the computer simulation technology has been suggested as a tool to study the complex system of recreation areas [16, 17], since it allows management to evaluate a change without going through the traumatic experience of making users change procedures before the results are known [18].

To obtain a more thorough understanding of and manage for complex interactions among tourists, models that systematically and precisely specify the interrelationships variables are required. However, it's difficult to develop such models since the recreation areas consist of lots of autonomous entities, besides; the entities are distributed over a large area and interact with each other. To conquer these obstacles, Wahle proposed that the characteristics of agent is well suited for the description of tourists [9]. They have respective mobility and cognitive capabilities, each of them collect information from the environment and make decisions, change their behaviour to achieve a specific goal. For this reason, Gimblett et al. [15] claimed that agent-based simulation had many advantages compared with those previously applied.

With the characteristics of tourists into consideration, this work tries to model the impact of real-time information on alleviating congestion in recreation areas using an agent-based model. Here, we must pay attention to the fact that the provision of information can give agents guidelines on how to make decisions rather than enforce agents to make any decision. In this work, we assume that all the agents behave "rationally", they trust the information they received and make the best decision based on all the information when face multi-choices.

\section{SIMULATION EXPERIMENTAL DESIGN}

In this study, an agent-based simulation is introduced for analysing such complex phenomena. It is known to be a powerful tool for visualizing such complex group dynamics and aids us to understand how each agent's decision affects total congestion phenomena as a result. For discussion on the congestion phenomena, it's necessary to delimit the properties of agents and the system from the mathematical point of view.

\subsection{Mathematical definition}

Let $A=\left[A_{1}, A_{2}, \ldots, A_{n}\right]$ be the set of agents while $B=\left[B_{1}, B_{2}, \ldots, B_{m}\right]$ be the set of attractions, $A_{i}$ represents agent $i$ while $B_{j}$ denotes attraction $j$. If agent $A_{i}$ visits the attraction $B_{j}$, then $x_{i j}=1$, else $x_{i j}=0 . t a_{i j}$ and $t e_{i j}$ represent the $A_{i}$ 's arrival time and departure time of $B_{j}$, respectively. It's often seen in recreation areas that the agents may have to wait when they arrive at attractions. Consequently, it's necessary to define another variable $t s_{i j}$, which denotes the $A_{i}$ 's start time of receiving service or sighting in $B_{j}$. It's evident that the $A_{i}$ 's wait time in $B_{j}$ is defined as 
$t w_{i j}=t s_{i j}-t a_{i j}$, and the total wait time of $A_{i}$ in the system is the sum of $t w_{i j}$, which can be calculated according to Eq. (1):

$$
T W_{i}=\sum_{j=1}^{m} x_{i j} \cdot t w_{i j}
$$

In addition, $W$ List $t_{i}$ stores the attractions that $A_{i}$ wishes to visit, while the $U_{n L i s t}$ stores the attraction it has not visited yet. In this paper, we assume that all the tourists wish to visit all the attractions, therefore, WList $t_{i}$ as the list contains all the attractions. Initially, the agent $A_{i}$ has not visited any of the attractions, thence, UnList $t_{i}$ equals to WList ${ }_{i}$.

Let $c_{j}$ represents the capacity of $B_{j}$, the service time in $B_{j}$ follow a normal distribution $N\left(t_{j}, s_{j}\right)$, where $t_{j}$ and $s_{j}$ express the means and standard deviation $(S D)$ of service time in $B_{j}$, respectively. Let $N_{j}^{t}$ represents the number of agents who are being serviced in $B_{j}$ at moment $t$, while $q_{j}^{t}$ denotes the number of waiting agents $(N W A)$. Also, $t m_{j k}$ denotes the moving time between $B_{j}$ and $B_{k}$. To simplify the problem, it assumes all the agents moving at the same velocity, which equals to one distance unit per minute. The distance between $B_{j}$ and $B_{k}$ is the length of the line segment connecting them, denoted as $d\left(B_{k}, B_{j}\right)$.

$z_{i j k}$ represents the state whether agent $i$ has moved from $B_{j}$ to $B_{k}$ or not, the value is initially 0 , and it's set to 1 if the agent moved from $B_{j}$ to $B_{k}$. Then the total moving time of agent $i$ can be given in Eq. (2), where $t m_{j k}$ represents the moving time from $B_{j}$ to $B_{k}$.

$$
T M_{i}=\sum_{j=1}^{m} \sum_{k=1 ; k \neq j}^{m} t m_{j k} \cdot z_{i j k}
$$

It is conceivable that all the agents achieve the goal of minimization unvalued time, which including wait time and moving time. The average unvalued time is defined as Eq. (3), where $n$ represents the total number of agents.

$$
A U V T=\frac{\sum_{i=1}^{n} T W_{i}+\sum_{i=1}^{n} T M_{i}}{n}
$$

The recreation managers have the responsibility to increase the agents' experiences as well as optimize the level of use in recreation areas. To achieve this goal, it's necessary to avoid excessive spatial concentration of agents in several attractions while at the same time other attractions are vacant. The $S D$ (Standard Deviation) of $N W A$ among attractions can evaluate the geographical distribution of agents. The average $N W A$ at $t$ moment can be formulated by Eq. (5), where $m$ denotes the number of attractions.

$$
\overline{q^{t}}=\frac{q_{1}^{t}+q_{m}^{t}+\cdots+q_{m}^{t}}{m}=\frac{\sum_{j=1}^{m} q_{j}^{t}}{m}
$$

The average standard deviation among attractions at $t$ moment $\left(s d^{t}\right)$ is calculated according to Eq. (6) and the average standard deviation among the process (ASD) is shown in Eq. (7), where $T$ means the total simulation steps. The $A U V T$ and $A S D$ are two important indicators to evaluate the strategies, which will be discussed later.

$$
\begin{gathered}
s d^{t}=\sqrt{\frac{\left(q_{1}^{t}-\overline{q^{t}}\right)^{2}+\left(q_{2}^{t}-\overline{q^{t}}\right)^{2}+\cdots+\left(q_{m}^{t}-\overline{q^{t}}\right)^{2}}{m-1}}=\sqrt{\frac{\sum_{j=1}^{m}\left(q_{j}^{t}-\overline{q^{t}}\right)^{2}}{m-1}} \\
A S D=\frac{s d^{1}+s d^{2}+\cdots+s d^{t}}{T}=\frac{\sum_{t=1}^{T} s d^{t}}{T}
\end{gathered}
$$




\subsection{Simulation scenarios description}

\section{a) Simulation parameter settings}

For the purpose of illustrating the impact of real-time on reduction congestion, a simulation situation with 9 attractions and 3 gates will be introduced, shown in Fig. 1. Suppose that all the attractions with the same value of $c_{j}$, $t_{j}$ and $s_{j}$, i.e., $c_{j}=50, t_{j}=15$ and $s_{j}=2$.

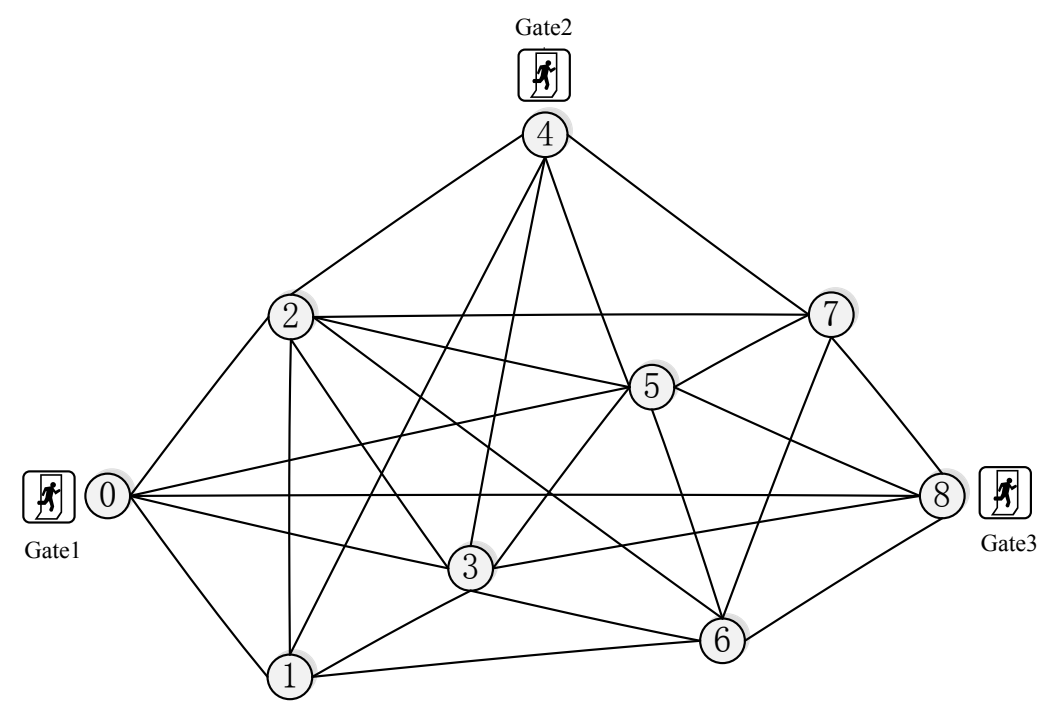

Figure 1: Example situation of simulation.

In this paper, we assume that the arrival distribution follow the Poisson distribution, since we notice the fact that the applications of the Poisson distribution are so popular in many fields related to counting, i.e., cars arriving, customers arriving, etc. [19]. Furthermore, in order to study the impact of real-time information on congestion situation, it's necessary to decrease or even eliminate the impacts caused by external factors. Therefore, we control the other external variables, i.e., the total number of agents and the total arrival times of agents, subject to the realistic description of agents' arrival.

\section{b) Simulation process}

Graphically the simulation process is represented as in Fig. 2. The process contains three stages, which will be described as follows:

(1) Setup Stage: In this stage, the first task is importing attractions' attributes, i.e., $c_{j}, t_{j}$, $s_{j}$, etc.; the other task is to generate the arrival-list of agents.

(2) Initial Stage: As described above, if the simulation steps $t$ no more than the total arrival times $\delta$, then $Y_{t}$ agents will enter the system, and thus have to choose among the three gates randomly.

(3) Steady Stage: This stage is the core of the simulation process, it contains two tasks: update-status and enter-attractions.

(3-1) Update-Status: Several statuses of agents are defined in the simulation model, including "initial" , "moving", "waiting", "service", "finish" and "departure", they represent different statuses of agents, respectively, shown as follows, and graphically the process of update-status is represented as in Fig. 3.

"Initial": the agent who just enters the gates, they will select one of attractions as their destination according to different rules, discussed later. After that, they will move to the destination and their status will become "Moving". 
"Moving": the agent who is moving from one attraction to another one. They will keep the status until they enter the queue of the destination, then the status change into "Waiting".

"Waiting": the agent who is waiting in the queue since their destinations operate at full capacity, they are allowed to enter the attraction follow the rule of first-in-first-out (FIFO) when the attraction below capacity, till then their status will turn into "Service".

"Service": the agents being service, its status will set to "Finish" when the simulation steps exceed their departure time, and then they will leave the attraction.

"Finish": the agents who just complete the service in an attraction, they have to choose between the two alternatives. If its set of UnList $t_{i}$ is empty, then it will leave the system and its status will change into "Departure", the number of agents who have completed to visit all of the attractions will be increased by 1 ; to the contrary, if its UnList $t_{i}$ isn't empty, then it will select one of attractions as their destination according corresponding rules.

"Departure": the agents who have completed to visit all of the attractions.

(3-2) Enter-Attractions: If the attraction $B_{j}$ doesn't operate at full capacity and nobody waiting in its queue, then the agent can enter $B_{j}$ directly. Otherwise, it enters the attraction follow the rule of first-in-first-out $(F I F O)$. To illustrate this process distinctly, $Q L_{i j}$ is defined to store the agent $A_{k}$ who waiting in the queue of $B_{j}$, and satisfying the condition: $t a_{k j}<t a_{i j}$. Then the number of agents in $Q L_{i j}$ is denoted as card $\left(Q L_{i j}\right)$. The process of Enter-attractions is given in Fig. 4.

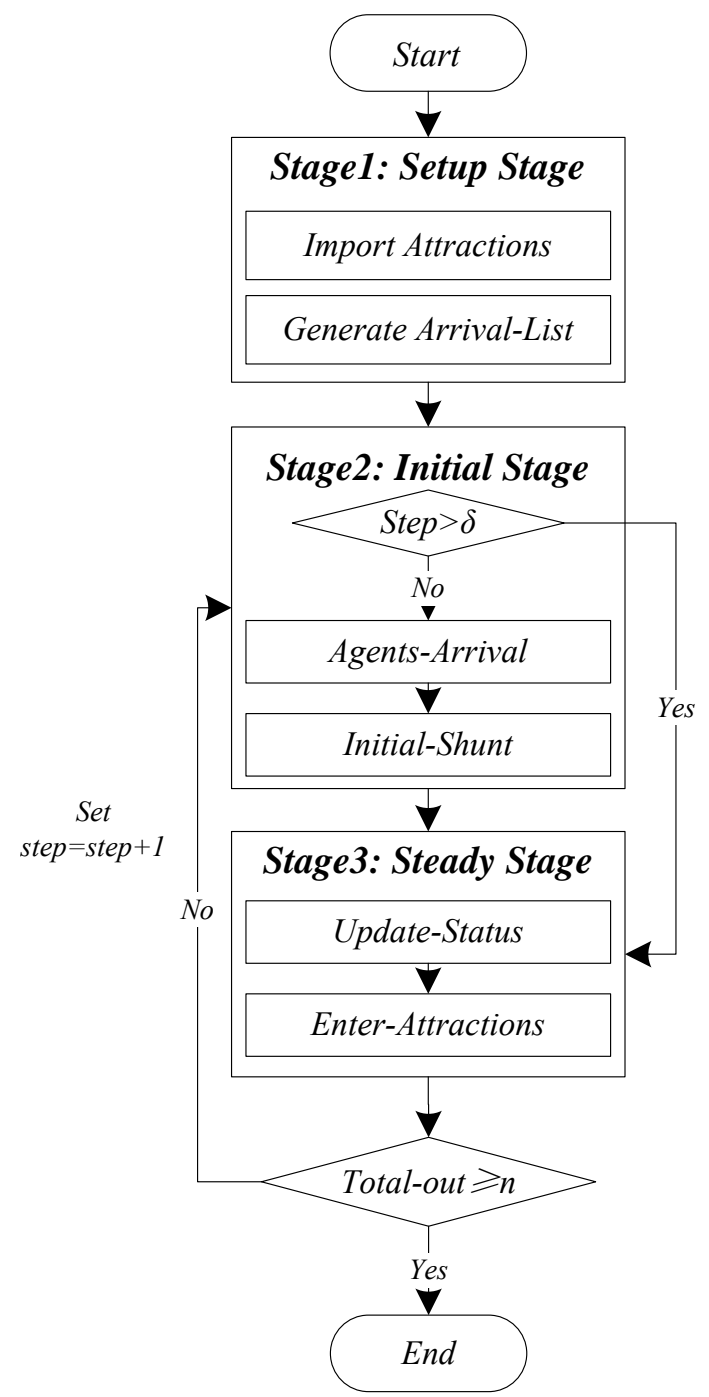

Figure 2: Flow chart of simulation process. 


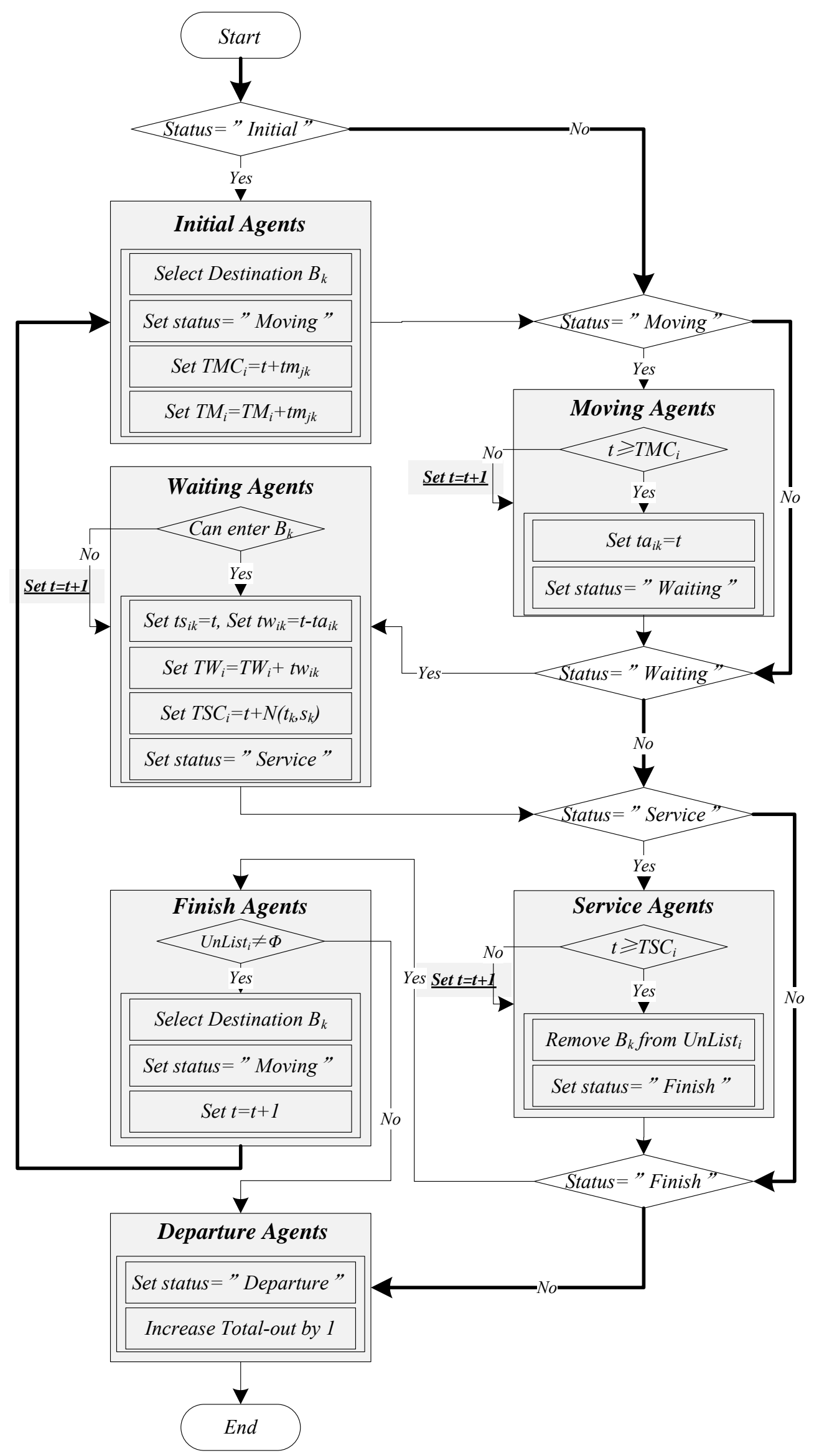

Figure 3: Process of Update-Status. 


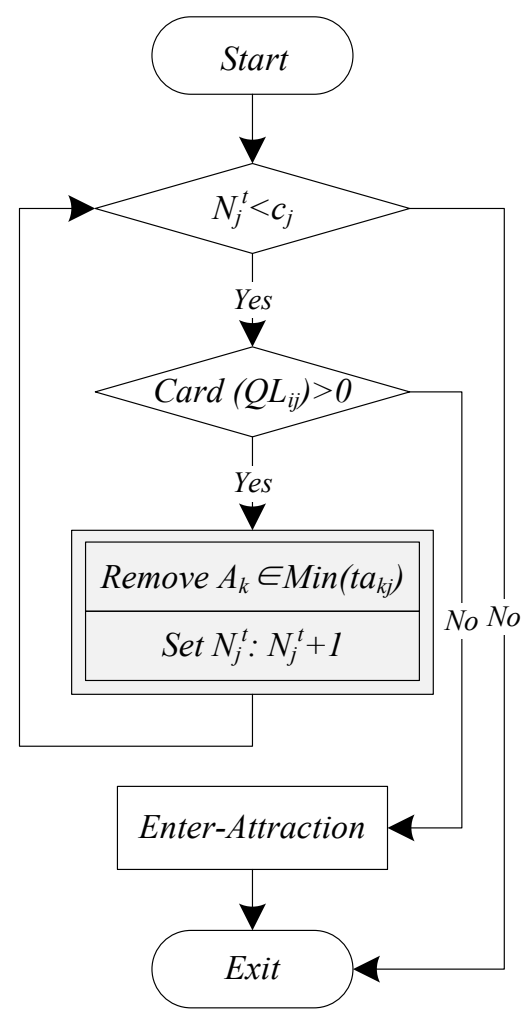

Figure 4: Process of Enter-Attractions.

\subsection{Information provision strategies}

In order to confirm the impact of information on congestion situation, three types of information provision strategies are identified firstly in this section: random strategy (RS), static strategy (SS) and dynamic strategy (RS).

\section{a) Random Strategy}

Generally the tourists tend to visits the attraction follow different sequences, since they have different preferences or perceive the situation in different ways. In this strategy, we suppose that the preferences of all agents are wholly different, and they haven't any information about the recreation areas; therefore, they have to select the destination randomly among the UnList $_{i}$. It's an extreme ideal situation, which leads to a natural distribution among the areas.

\section{b) Static Strategy}

In the static strategy, the static information regarding the distance between attractions is sent to the agents. When they finished the visitation at the current attraction, the agents head for the nearest destination from the remaining attractions to visit on the basis of static information, e.g. the moving time from the current attraction to other attraction among the UnList. The agent can acquire the static information from the area map or the signs in the area. It heads for the nearest attraction from its current position in line distance. The selection rule can be described as Eq. (8):

$$
B_{d e s}=\left\{k \mid t_{j k}=\operatorname{Min}\left(t_{j k}\right), k \in U n L i s t_{i}\right\}
$$

\section{c) Dynamic Strategy}

The dynamic information includes all the static information, moreover, some real-time information, such as service time and the waiting number for each attraction is presented so that the agents can determine the visit sequence. The agent receives the dynamic information through information equipment such as PDA, then it optimizes its trip, i.e., it selects the attraction with the minimal-visit-cost. If the information for several attractions is the same, an 
attraction is chosen randomly. The visit-cost, including moving time and estimated wait time, can be calculated according to Eqs. (9) and (10), where $E W T_{j k}{ }^{t}$ denotes the estimated wait time of $B_{k}$ at $t$ moment, while $M C_{j k}{ }^{t}$ represents the visit cost from $B_{j}$ to $B_{k}$ at $t$ moment. Then the selection rule based on minimal-visit-cost formulated as Eq. (11).

$$
\begin{gathered}
E W T_{j k}^{t}=\left\{\begin{array}{l}
0, q_{k}^{t}+N_{k}^{t}<c_{j} \\
\left(q_{k}^{t}+N_{k}^{t} / 2\right) \times t_{k} / c_{k}, \text { else }
\end{array}\right. \\
M C_{j k}^{t}=E W T_{j k}^{t}+t m_{j k} \\
B_{d e s}=\left\{k \mid M C_{j \rightarrow k}=\operatorname{Min}\left(M C_{j \rightarrow k}\right), k \in \text { UnList }_{i}\right\}
\end{gathered}
$$

\section{SIMULATION RESULTS AND DISCUSSION}

\subsection{Simulation results}

In the "random strategy", the agents determine the destinations since no information is shared among them. Randomly generating the destination for each agent could contribute to balance the geographical distribution of agents and avoid the concentration of agents in certain attractions [20]. Therefore, the $N W A$ at each attraction is relatively small, as shown in Fig. 5, where horizontal axis shows the simulation step and each curve shows the NWA (agents in waiting mode) at each attraction (the curve " $B_{j}$ " corresponds to the attraction $B_{j}$ ).

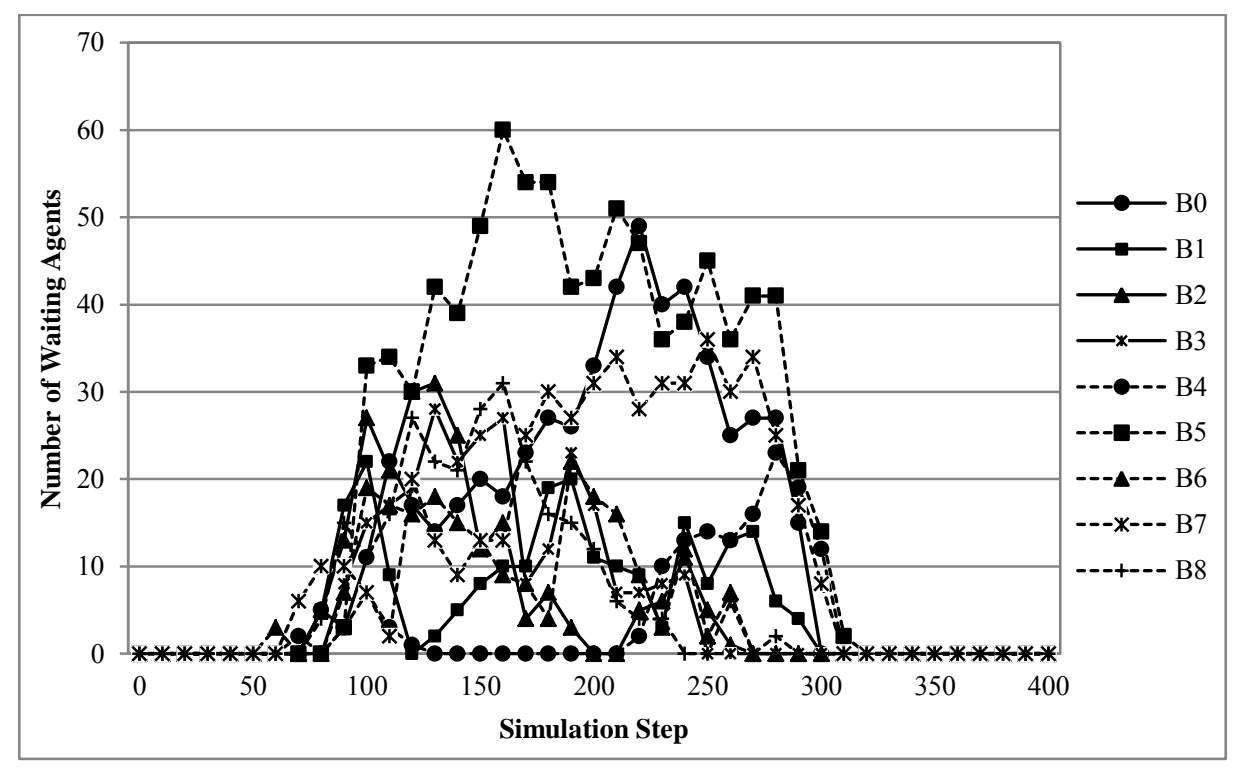

Figure 5: The $N W A$ at each attraction (random strategy).

Compared with random strategy, amplitude of the oscillations appears in the static strategy, displayed in Fig. 6. It's necessary to investigate the simulation discipline of this strategy, since it will conduce to reveal the essence of oscillations. In this strategy, the agents enter into the areas from one of the gates randomly, and head for their destinations, and then the NWA increased at all attractions. According to the selection rule of static strategy and the actual distance between attractions, the agents from gate 1 will visit the areas follow the sequence [0-1-3-5-7-8-6-4-2], while the agents from other two gates will follow the orders [45-7-8-6-3-1-0-2] and [8-7-5-3-1-0-2-4-6], separately. It is evident that all the agents visit $B_{5}$ and $B_{4}$ in the primary phase of the tour while visit $B_{2}$ near-final stage, leading to the great range in fluctuations of waiting number of $B_{5}, B_{4}$ and $B_{2}$, as shown in Fig. 6. 


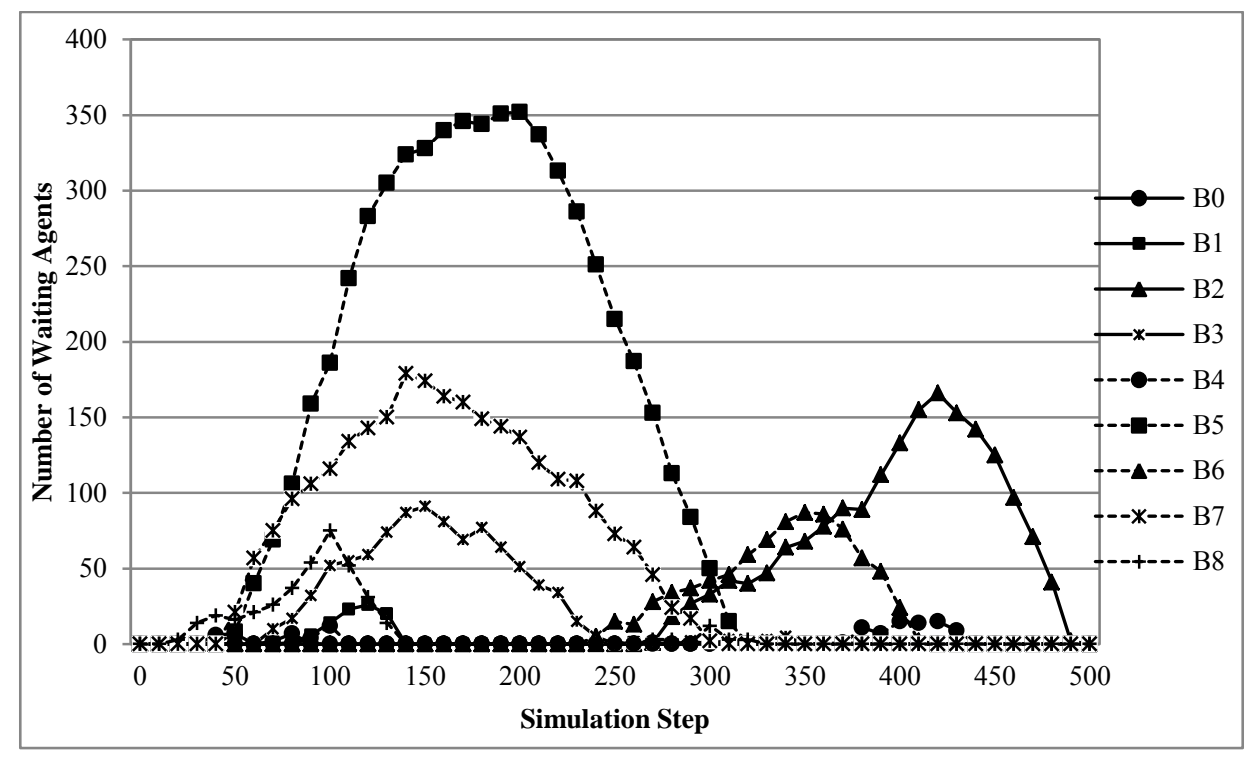

Figure 6: The NWA at each attraction (static strategy).

In Fig. 7, it can be seen that each attraction experiences a similar fluctuation in dynamic strategy, and the crests are more dispersed compared with other strategies.

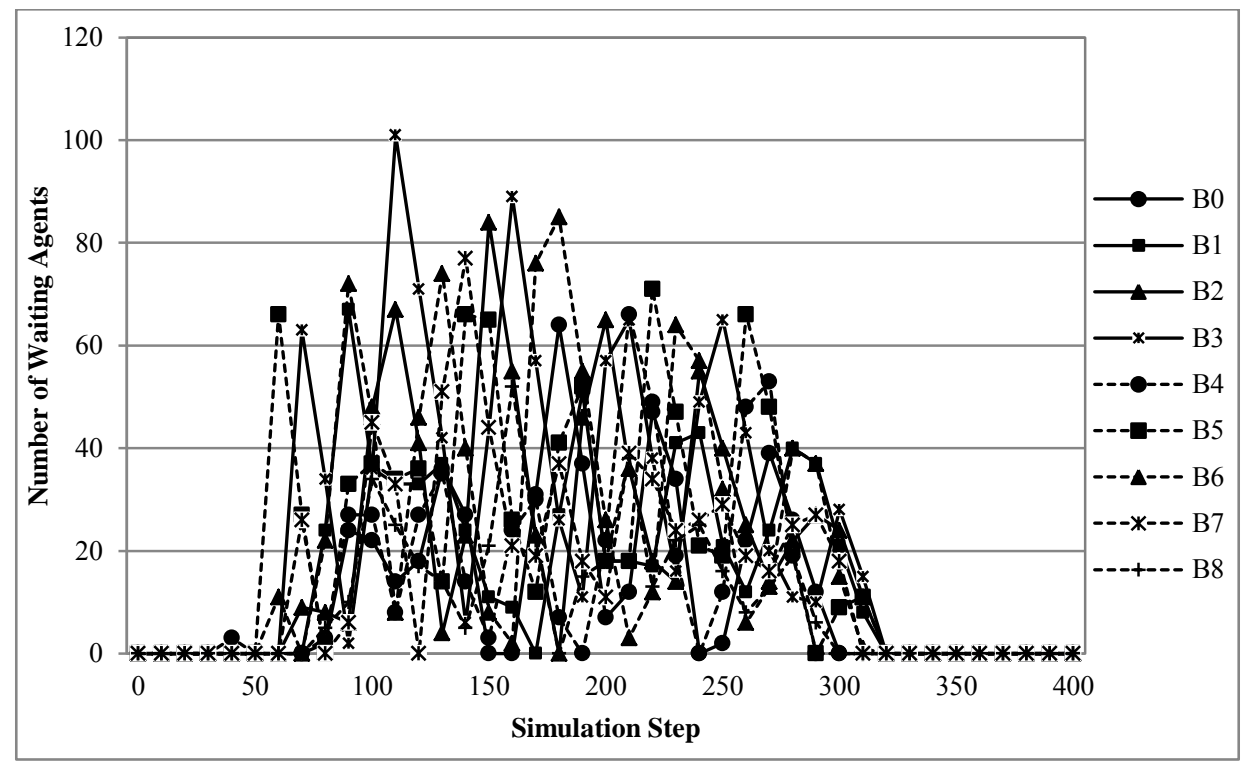

Figure 7: The NWA at each attraction (dynamic strategy).

By comparing the $N W A$ at each attraction, the equilibrium of random and dynamic strategy far exceed that of static strategy. The value of $A S D$ which was defined before also reflects this result, 66.80 for static strategy, 11.73 for random strategy and 18.22 for dynamic strategy. The data indicates that the random strategy performs best, dynamic strategy comes second and static strategy brings up the rear from the angle of agents' balanced distribution.

As for the comparison of agents' time-consuming, which includes average wait time $(A W T)$, average moving time $(A M T)$ and average unvalued time $(A U V T)$ is displayed in Fig. 8. As described above, the largest oscillation of $N W A$ in static strategy leads to its maximum of $A W T$, peaking at 137.33 minutes, which is almost over three times than that of random strategy and double than that of the dynamic strategy. By contrast, the static strategy achieves the least of $A M T$ because of its selection rule, while the agents of random strategy spend more 
time on moving, compared with the other two strategies. As for $A U V T$, which is the total of $A M T$ and $A W T$, it's clear that the most of $A U V T$ is static strategy, and the value of random strategy is slightly more than that of dynamic strategy.

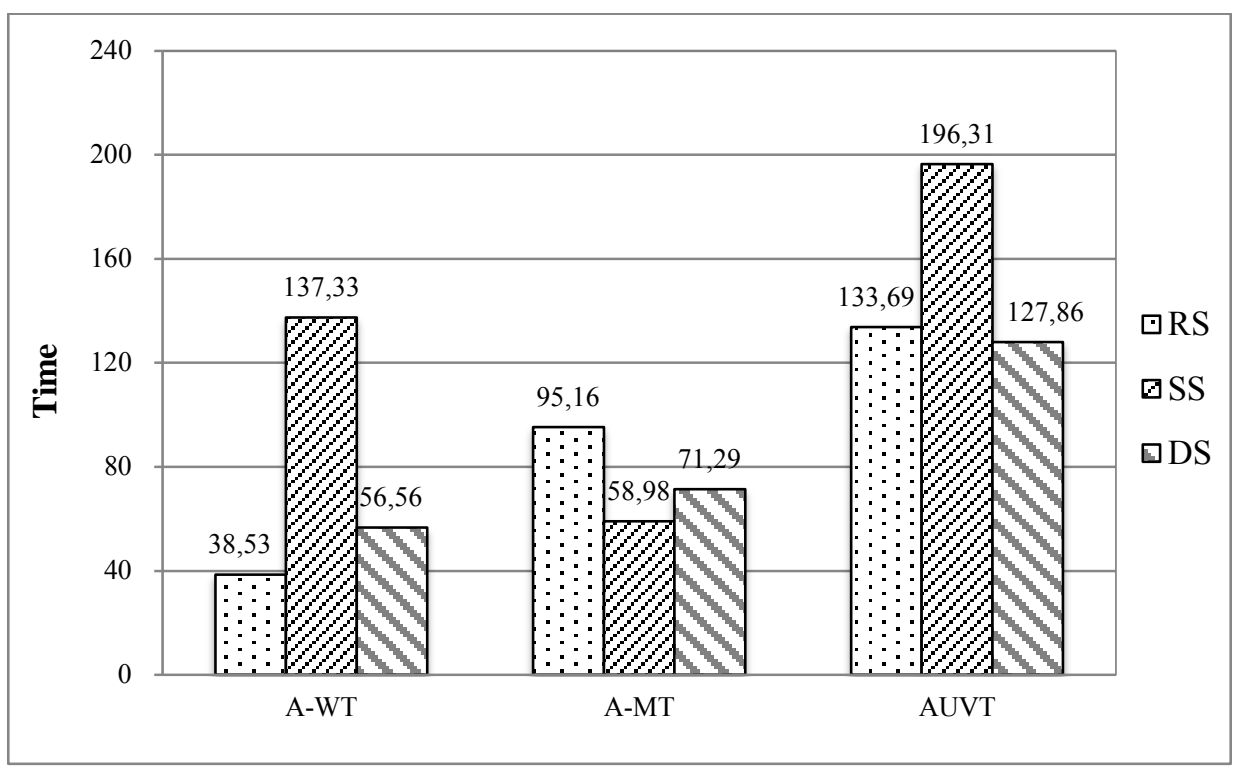

Figure 8: Comparison of agents' time-consuming.

\subsection{Discussion}

A dramatic phenomenon can be found that may different with the traditional acknowledge through the comparison. It seems that the more information shared among the agents, the more likely to relieve congestion, whereas, the different results generated in the simulation experiments. In this section, we discuss the reason for this reason.

A set of agents who enter from one entrance tend to select different visiting sequences, since they have different preferences, this leads to a natural distribution among the recreation areas, and then reduce the number of waiting agents at each attraction. Sharing information can reduce this variation if all the agents receive the same information. In particular, in the static information sharing pattern, the static information prompts the agents who enter from the same entrance to choose the same visiting sequence. Therefore, the $A-W T$ and $A S D$ of this strategy are significantly higher than the other two strategies. In the dynamic sharing pattern, the agents who receive the real-time information continually head for the destination with minimal-visit-cost within a certain time frame despite the fact that some agents are already moving toward that attraction since the attraction remain the best alternative until agents begin arriving there. Since every agent behaves in the same way, the attraction with minimalvisit-cost is selected more often. Therefore, that attraction becomes overcrowded due to excessive agent concentration. Another attraction then becomes the optimal choice, and the cycle repeats. Then the queue length at each attraction thus oscillates. The congestion situation of this strategy is more superior to that of static strategy $(A-W T: 56.56<137.33$; the $A S D: 18.22<66.80)$, but it still slightly inferior to random strategy $(A W T: 56.56>38.53$; $A S D: 18.22>11.73)$. So far as waiting time and distribution are concerned, it's better not to share any information to agents. On the other hand, sharing information can reduce the blindness in visiting the recreation areas. As shown above, without information, the average moving time is significantly longer.

All in all, when exploring the information provision to reduce the congestion, both of its potential benefits as well as its drawbacks should be taken into consideration. 


\section{CONCLUSION}

The impact of information sharing on reduction congestion in recreation areas is studied using a simplified model of a tourist as an agent in a multi-agent model and applying it to abstract destination-choice scenario. Several issues are revealed in this work: Firstly, sharing information among tourists has an enormous impact on congestion in recreation areas, and the direction and degree of impact depend upon the types of information provision. Secondly, information sharing can bring side effects, such as concentration and congestion oscillation, while real-time information can reduce these negative effects.

The potential benefits and drawbacks of information provision on relieving congestion in recreation areas may be discussed continuously in the future, however, we believe that the study on the impact of information sharing on congestion would not only deepen the understanding of the collective behaviour of tourists but also could enhance the management in tourism and traffic domain. As a next step in our analysis, future research might address the investigation for the impact of information on different types of tourists and formulate the corresponding information sharing patterns according to the tourists.

\section{ACKNOWLEDGEMENTS}

This work was supported by the Major International Joint Research Program of the National Natural Science Foundation of China (Grant No. 71020107027), the National Natural Science Foundation of China (Grant No. 71001075), the scholarship from China Scholarship Council (No.201306240069), and Central University Fund of Sichuan University (No. skqy201112).

\section{REFERENCES}

[1] Li, M.; Wu, B.; Cai, L. (2008). Tourism development of World Heritage Sites in China: A geographic perspective, Tourism Management, Vol. 29, No. 2, 308-319, doi:10.1016/j.tourman. 2007.03.013

[2] Cui, X.; Ryan, C. (2011). Perceptions of place, modernity and the impacts of tourism Differences among rural and urban residents of Ankang, China: A likelihood ratio analysis, Tourism Management, Vol. 32, No. 3, 604-615, doi:10.1016/j.tourman.2010.05.012

[3] Jakus, P.; Shaw, W. D. (1997). Congestion at recreation areas: empirical evidence on perceptions, mitigating behaviour and management preferences, Journal of Environmental Management, Vol. 50, No. 4, 389-401, doi:10.1006/jema.1997.0124

[4] Alegre, J.; Cladera, M. (2006). Repeat visitation in mature sun and sand holiday destinations, Journal of Travel Research, Vol. 44, No. 3, 288-297, doi:10.1177/0047287505279005

[5] Bhadra, D. (2009). You (expect to) get what you pay for: A system approach to delay, fare, and complaints, Transportation Research Part A: Policy and Practice, Vol. 43, No. 9-10, 829-843, doi:10.1016/j.tra.2009.08.003

[6] Tsai, C.-Y.; Chung, S.-H. (2012). A personalized route recommendation service for theme parks using RFID information and tourist behavior, Decision Support Systems, Vol. 52, No. 2, 514-527, doi:10.1016/j.dss.2011.10.013

[7] Kawamura, H.; Kataoka, T.; Kurumatani, K.; Ohuchi, A. (2004). Investigation of global performance affected by congestion avoiding behavior in theme park problem, IEEJ Transactions on Electronics, Information and Systems, Vol. 124, No. 10, 1922-1929, doi:10.1541/ieejeiss. $\underline{124.1922}$

[8] Hogg, T.; Huberman, B. A. (1991). Controlling chaos in distributed systems, IEEE Transactions on Systems, Man and Cybernetics, Vol. 21, No. 6, 1325-1332, doi:10.1109/21.135679

[9] Wahle, J.; Bazzan, A. L. C.; Klugl, F.; Schreckenberg, M. (2002). The impact of real-time information in a two-route scenario using agent-based simulation, Transportation Research Part C: Emerging Technologies, Vol. 10, No. 5-6, 399-417, doi:10.1016/S0968-090X(02)00031-1 
[10] Ben-Akiva, M.; De Palma, A.; Isam, K. (1991). Dynamic network models and driver information systems, Transportation Research Part A: General, Vol. 25, No. 5, 251-266, doi:10.1016/01912607(91)90142-D

[11] Suzuki, R.; Arita. T. (2003). Effects of information sharing on collective behaviors in competitive populations, Proceedings of the Eighth International Symposium on Artificial Life and Robotics $(A R O B)$, Oita, 36-39

[12] Kataoka, T.; Kawamura, H.; Kurumatani, K.; Ohuchi, A. (2005). Distributed visitors coordination system in theme park problem, Ishida, T.; Gasser, L.; Nakashima, H. (Eds.), Massively MultiAgent Systems I, Springer-Verlag, Berlin, 335-348, doi:10.1007/11512073 25

[13] Dia, H. (2002). An agent-based approach to modelling driver route choice behaviour under the influence of real-time information, Transportation Research Part C: Emerging Technologies, Vol. 10, No. 5-6, 331-349, doi:10.1016/S0968-090X(02)00025-6

[14] Potter III, F. I.; Manning, R. E. (1984). Application of the wilderness travel simulation model to the Appalachian Trail in Vermont, Environmental Management, Vol. 8, No. 6, 543-550, doi:10.1007/BF01871580

[15] Gimblett, R.; Daniel, T.; Meitner, M. J. (2000). An individual-based modeling approach to simulating recreation use in wilderness settings, USDA Forest Service Proceedings RMRS-P-15VOL-4, Missoula, 99-106

[16] Cesario, F. J. (1975). A simulation approach to outdoor recreation planning, Journal of Leisure Research, Vol. 7, No. 1, 38-52

[17] Romesburg, C. (1974). Scheduling models for wilderness recreation, Journal of Environmental Management, Vol. 2, No. 2, 159-177

[18] Underhill, A. H.; Xaba, A. B.; Borkan, R. E. (1986). The wilderness use simulation model applied to Colorado River boating in Grand Canyon National Park, USA, Environmental Management, Vol. 10, No. 3, 367-374, doi:10.1007/BF01867261

[19] Rasch, G. (1963). The Poisson process as a model for a diversity of behavioral phenomena, International Congress of Psychology, Washington DC

[20] Li, J.; Zhu, D. (2009). Multi-stage dynamic coordination and control model for mass crowds activities based on multi-agent, Chinese Journal of Management Science, Vol. 17, No. 5, 113 119 